\title{
Efficacy and Tolerability of Injectable Collagen-Containing Products in Comparison to Trimecaine in Patients With Acute Lumbar Spine Pain (Study FUTURE-MD-Back Pain)
}

\author{
K. PAVELKA ${ }^{1}$, H. JAROSOVA ${ }^{1}$, L. MILANI ${ }^{2,3}$, Z. PROCHAZKA $^{4}$, P. KOSTIUK ${ }^{4}$, \\ L. KOTLAROVA ${ }^{5}$, A. M. MERONI ${ }^{6}$, J. SLIVA $^{7}$
}

${ }^{1}$ Institute of Rheumatology, Prague, Czech Republic, ${ }^{2}$ University Sapienza, Rome, Italy, ${ }^{3}$ University of Siena, Italy, ${ }^{4}$ Edukafarm, Prague, Czech Republic, ${ }^{5}$ Department of Pharmacology, InpharmClinic, Jesenice u Prahy, Czech Republic, ${ }^{6}$ Department of Orthopedics and Traumatology, Niguarda Hospital, Milano, Italy, ${ }^{7}$ Third Faculty of Medicine, Charles University, Prague, Czech Republic

Received August 22, 2019

Accepted September 4, 2019

\begin{abstract}
Summary
Low back pain (LBP) represents an important subgroup of vertebrogenic pain with estimated prevalence around $80 \%$. Locally acting injectable collagen for topical application has recently extended the limited range of treatment options. The aim of the study was to evaluate the efficacy and safety of injectable collagen in patients with LBP. Patients suffering from LBP ( $<$ three months) were enrolled. They were administered either collagen $4 \mathrm{ml}$ or trimecaine $1 \% 4 \mathrm{ml}$ in the form of subcutaneous paravertebral injections into eight pre-specified points ( $0.5 \mathrm{ml}$ per each point) in the following schedule: two administrations in the first and second week, one in the third week. The pain intensity, Thomayer distance, Oswestry disability index, Lasseque test, quality of life, consumption of rescue medication and safety were evaluated. Exertional and rest pain, evaluated by a visual analogue scale, gradually decreased in both groups. Both treatments showed a statistically significant improvement in mobility and quality of life. The consumption of paracetamol as a rescue medication was significantly lower in patients treated with collagen than in the group treated with trimecaine $(p=0.048)$. The analgesic efficacy of locally acting injectable collagen, as well as an analgesic sparing effect when compared to local anesthetics were demonstrated.
\end{abstract}

\section{Key words}

Low back pain • Injectable collagen • Analgesics • Paracetamol • Trimecaine • Pain

\section{Corresponding author}

J. Slíva, Department of Pharmacology, Third Faculty of Medicine, Charles University, Ruská 87, 10000 Prague 10, Czech Republic. E-mail: Jiri.Sliva@If3.cuni.cz

\section{Introduction}

Musculoskeletal pain including vertebrogenic pain is among the most common health complaints with significant social and economic consequences. The incidence of musculoskeletal pain increases with the rising average age of the population and decreases the quality of life of a growing number of affected people. Back pain represents an important subgroup of vertebrogenic pain. It is reported that approximately $80 \%$ of the population in developed countries have back pain problems for which they seek medical help, at least once in a lifetime (NICE 2016).

Back pain can be classified according to its localization as neck pain (NP) or low back pain (LBP). Pain/discomfort affecting the area from the lower rib margin to the lower gluteal fold is called LBP. Lower limb pain may or may not be present at the same time. LBP can be classified by its duration as acute (back pain episodes lasting no more than three months, sometimes a subgroup of subacute pain lasting 6 to 12 weeks is also distinguished) or chronic (when pain lasts more than three months). The recurrence of problems after at least 
a six-month symptom free period is known as recurrent LBP (this form must be distinguished from chronic LBP exacerbation). According to the etiology, LBP is classified as specific when the pathological process causing the pain is known (e.g. inflammatory or degenerative process, fracture, radicular syndrome, etc.) or nonspecific (when such a causative process is not apparent).

Patients with acute back pain which subsides within six weeks to three months represent the majority of the affected people. However, approximately $2-7 \%$ of patients move to the group of chronic pain that can seriously affect not only health, but due to limited working ability, also the social and economic aspects of a patient's life. This type of chronic pain, due to back disorders, contributes to the extent of $90 \%$ of social costs of sick leave. Concerning back pain distribution according to age groups, elderly patients are affected more often. In younger people, back pain typically arises from excessive burden to normal spinal structures. With advancing age, pathological processes (i.e. processes of degenerative nature) play an increasing role in the etiology. The symptoms and morphological findings of LBP have a very weak correlation and the pain is not always proportional to the pathological changes.

The patient's history and neurological examination play important diagnostic roles. If severe spinal pathology is suspected (specific biomedical factors or so-called "red flags", e.g. root syndromes, fractures and infections), imaging techniques are indicated to specify the cause (e.g. X-ray, magnetic resonance imaging [MRI]). Psychosocial factors (so-called "yellow flags") also play an important role and increase the risk of problem chronification. If no specific causative factors are presented, the condition is called nonspecific LBP (Nice 2016).

The problem of back pain treatment is complex due to the existence of many well-known, but also some unknown pathophysiological factors that make treatment difficult. The ranges of medical procedures include a multidisciplinary approach with conservative methods to minimally invasive interventions to surgical treatment. Conservative procedures in acute nonspecific LBP include mainly rehabilitation and medications (NICE 2016, van Tulder et al. 2006). These non-invasive treatment methods are effective in 80 to $90 \%$ of patients with LBP and therefore, conservative treatment is considered the method of choice for most patients with back pain syndromes. In the treatment of acute nonspecific LBP, paracetamol is recommended as the first-line treatment and nonsteroidal anti-inflammatory drugs (NSAIDs) or muscle relaxants are recommended as the second-line treatment. In some cases, acupuncture is used; however, today it is believed to be suitable only for chronic LBP (Furlan et al. 2005, Itoh et al. 2004) or a local anesthetic is applied. Collagen-containing products intended for topical application have also recently extended the limited range of treatment options (Pavelka et al. 2019). Other possible therapeutic approaches have been postulated. These include (for example) newer intradiscal therapies (Charneux et al. 2017, Knezevic et al. 2017), modulation of galanin receptors (Zhang et al. 2019), modulation of TRPA-1 (Liu et al. 2019, Yamamotova et al. 2017), monoclonal antibody tanezumab (Webb et al. 2018), etc. (Bhangare et al. 2017).

One of the major causes of musculoskeletal pain is weakness of the internal and external joint stabilization systems. The basic component of these systems is collagen, the sufficient content and quality of, which are essential for the intact function of these structures. A lack of collagen or failure of its composition leads to weakening of the support systems and joint hypermobility, especially in non-physiological positions. This leads to premature wear of these systems and further increases the risk of progressive degeneration of structures such as cartilage. Slack hypermobile elements of the support system stimulate pain receptors and lead to muscle tension around the joints (Milani 2010, Stone et al. 1997). On the basis of these findings, collagencontaining products for local administration (as medical devices generally known as MD-products) have been developed. These include the MD-Lumbar, MD-Neural and MD-Muscle products (Guna, Milano, Italy) used in our study. Patients with acute lumbar spine pain were enrolled if their condition required the administration of local anesthetics or collagen-containing products. This study evaluated the efficacy and safety of the MD-Lumbar, MD-Nural and MD-Muscle products in comparison to the subcutaneously administered local anesthetic trimecaine.

\section{Methods}

\section{Patients}

Adult outpatients of both sexes who met the diagnostic criteria for acute lumbar spine pain, defined as LBP lasting no more than three months, were enrolled in 
a prospective, single-blind clinical trial titled FUTUREMD-Back Pain. A total of 97 patients were enrolled and were randomized into two groups. Randomization was performed by a generator (available from www.randomization.com) using random permuted blocks (blocks of 20 subjects), with a randomization ratio of $3: 1$. All patients underwent in total seven visits

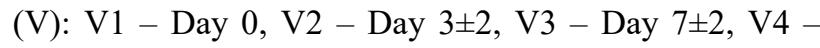
Day $11 \pm 2$, V5 - Day $14 \pm 2$, V6 - Day $18 \pm 2$, and V7 Day $32 \pm 2$.

\section{Materials and chemicals}

The study mixture of MD-Lumbar MD, MD-Muscle and MD-Neural ("A") was administered subcutaneously to the first group of patients aged 26-70 years ( $\mathrm{n}=73$, including 24 males and 49 females) and Mesocaine $1 \%$ (trimecaine hydrochloride) ("B") was subcutaneously administered to the second group aged 26-66 years $(\mathrm{n}=24,7 \mathrm{men}$ and 17 women). The combination of MD-Muscle (1 ml), MD-Lumbar $(2 \mathrm{ml})$ and MD-Neural $(1 \mathrm{ml})$ for a total of $4 \mathrm{ml}$ per dose or Mesocaine $1 \% 4 \mathrm{ml}$ per dose were administered in the form of subcutaneous paravertebral injections into eight pre-specified points $(0.5 \mathrm{ml}$ per one application point) for a total duration of three weeks. A total of five applications were made in each patient (twice the first week, twice the second week and once the third week). If a subject not completing the study was replaced, the new subject received the same product as the patient excluded from the clinical trial.

MD-Lumbar, MD-Neural and MD-Muscle are medical devices designed to improve mobility and reduce pain in the lumbar spine area. Collagen is the primary active ingredient of these combined products. The effect of topical collagen administration is structural and functional. Collagen is directly delivered to the area where it is lacking to strengthen, give structure and protect joint cartilage, tendons, ligaments and joint capsules by creating an adhesive barrier (Milani 2010). The transport of collagen along with the other ingredients to the local destination is based on a patented "collagen delivery system". When using this system, a temporary porous collagen matrix forms in the tissue where the product is applied, and gradually releases the active substances into the targeted area at a defined speed (depending on the porosity of the matrix), thus ensuring prolonged activity. The main therapeutic functions of collagen include the barrier effect, lubricating activity and support of potential concomitant analgesic medication. Collagen helps to improve the functionality of the joints, improves the profile of the collagen fibres and, consequently, all the anatomical collagen-containing structures. When strengthening the joint stabilization systems with locally administered collagen, an analgesic effect is achieved in addition to a structural recovery (creating biological support, so-called bioscaffold). The locally administered collagen in these products helps to relieve painful muscle tension in the region and restore its physiological function. Therefore, it helps to eliminate the cause of pain and to normalize the function of the affected joint and its supporting apparatus. The other ingredients of individual collagen-containing products differ (Randelli et al. 2018).

In addition to collagen, MD-Lumbar contains an extract from the bark and roots of the medicinal plant Hamamelis virginiana. The active ingredients of this extract are tannins, organic acids and essential oils; the extract has anti-inflammatory and antioxidant effects. It protects tissues at the injection site from the development of the inflammatory process that occur secondary to degenerative changes of the connective tissue; this inflammation further damages the tissues affected by degeneration. The anti-inflammatory effect of the extract from Hamamelis virginiana thus contributes to the effect of collagen. The extract, in addition to its antioxidative action, protects the integrity of tissues at the application site from the effects of harmful oxygen radicals.

MD-Neural is a medical device designed primarily to suppress neuropathic pain in different locations via its effect on the collagen component of the perineurium. In addition to collagen, it contains an extract from the medicinal plant Citrullus colocynthis. The extract from this plant is used in low concentration dilution in traditional medicine as a spasmolytic and analgesic. The analgesic effects of the ingredients (e.g. bitter compounds, triterpenes, and resins) are used in neuropathic pain, i.e. the stabbing pain typical for lumbago and sciatica.

MD-Muscle is a medical device which acts as an analgesic due to its effect on muscle tissue. In addition to collagen, it contains an extract from the medicinal plant Hypericum perforatum. Besides showing antidepressant action, this extract has anti-inflammatory and analgesic effects. It contains the full spectrum of active ingredients (e.g. hypericin, hyperforin, tannins, flavonoids) with anti-inflammatory, analgesic and regenerative effects. Its analgesic effects can be useful in cases of neuropathic and muscle pain.

Trimecaine (product Mesocaine $1 \%$ ) belongs to 
the group of medicines that produce local tissue anesthesia. It is an amide local anesthetic with a moderately long effect, which is rapidly and well absorbed. It inhibits genesis and conduction of painful stimuli and other centripetal impulses generated by pressure, tension, heat, etc. The anesthetic effect occurs within 15 min after administration and lasts for 60-90 min.

\section{Evaluation of effectiveness}

Both exertional and rest pain were measured by visual analogue scale (VAS) at each visit. Additionally, both Thomayer distance (expressed in $\mathrm{cm}$ ) and Lassegue test were evaluated on V1, V3, V6, and V7. A quality of life questionnaire (EuroQol) and Oswestry Disability Index were measured on V2, V6, and V7. During the whole treatment period (i.e. V1-V6), the total paracetamol consumption was evaluated. Finally, the tolerability of both treatments was assessed by the patients during V6 and V7.

\section{Statistical analysis}

Thomayer distance, Oswestry Disability index, EuroQol and VAS were evaluated using repeated measures analysis and post hoc Fisher's LSD (Least Significant Difference) test. Paracetamol consumption was evaluated using a one-tailed T-test. Treatment tolerability was assessed using the chi-square test.

\section{Results}

Patients were recruited for a total of about six weeks and a three day wash-out period was carried out, during which all analgesic and anti-inflammatory treatments were discontinued and patients were only allowed to use paracetamol. After the baseline examination, the evaluated products were administered for three weeks followed by a two week follow-up (for a total of 7 visits). The efficacy of the treatment was evaluated at individual visits. The tolerability of the treatment was assessed at the same time, both by the patient and by the attending physician. The current intensity of lumbar spine pain was measured by means of a horizontal visual analogue scale (VAS). The efficacy was also evaluated by identifying trigger points, evaluation of back muscle spasms, examination of the Thomayer distance and the Lassegue test. In addition, the EuroQol questionnaire (characterizing the quality of life of the patient) and the Oswestry questionnaire (Oswestry Disability Index) were also used for evaluation. The Oswestry questionnaire, which was completed at V2 and V6, quantifies the restriction of daily activities due to LBP, quantifies the subjective complaints of the patient and expresses the degree of disability, i.e. it reflects also the quality of life of the patient. The final examination was performed two weeks after the last treatment administration. The final examination (follow-up) included, besides the clinical examination, the overall efficacy of the treatment assessed by the physician and by the patient, as well as the patient's functional status. The patients again completed the final EuroQol and Oswestry questionnaires. The schematic design of the study is given in Table 1a.

Table 1a. Clinical study design.

\begin{tabular}{|c|c|c|c|c|c|c|c|}
\hline & Visit 1 & Visit 2 & Visit 3 & Visit 4 & Visit 5 & Visit 6 & $\begin{array}{c}\text { Visit } 7 \\
\text { (follow-up) }\end{array}$ \\
\hline Examınation & $\begin{array}{c}\text { Day } 0 \\
\text { Week } 0\end{array}$ & $\begin{array}{l}\text { Day } 3 \pm 2 \\
\text { Week 1a }\end{array}$ & $\begin{array}{r}\text { Day } 7 \pm 2 \\
\text { Week 1b }\end{array}$ & $\begin{array}{l}\text { Day } 11 \pm 2 \\
\text { Week } 2 \text { a }\end{array}$ & $\begin{array}{l}\text { Day } 14 \pm 2 \\
\text { Week 2b }\end{array}$ & $\begin{array}{c}\text { Day } 18 \pm 2 \\
\text { Week } 3\end{array}$ & $\begin{array}{c}\text { Day } 32 \pm 2 \\
\text { Week } 5\end{array}$ \\
\hline Baseline examinations & + & - & - & - & - & - & - \\
\hline Medical history & + & - & - & - & - & - & - \\
\hline Clinical examination & + & + & + & + & + & + & + \\
\hline Laboratory assessments & + & - & - & - & - & - & - \\
\hline$B P$ & + & + & + & + & + & + & + \\
\hline Current treatment & + & + & + & + & + & + & + \\
\hline Product administration & - & + & + & + & + & + & - \\
\hline $\begin{array}{l}V A S-\text { evaluation of efficacy by } \\
\text { the patient, by the physician }\end{array}$ & + & + & + & + & + & + & + \\
\hline EuroQol questionnaire & - & + & - & - & - & + & + \\
\hline
\end{tabular}


Table 1b. Characteristics of the population.

\begin{tabular}{lcc}
\hline Item & MD inj. & Mesocaine inj. \\
\hline $\begin{array}{l}\text { Number of patients } \\
\text { - men }\end{array}$ & 24 & 7 \\
- women & 49 & 17 \\
- total & 73 & 24 \\
Patient age (years) & & \\
- men & $48.1 \pm 12.8$ & $46.4 \pm 13.6$ \\
- women & $55.0 \pm 10.7$ & $56.6 \pm 10.0$ \\
Analgesic & & \\
treatment before & $42 / 31(57.5 \%)$ & $14 / 10(58.3 \%)$ \\
the YES/NO & & \\
\end{tabular}

* - statistically significant difference from baseline $p<0.05$ (MANOVA and post hoc Fisher's LSD test). The difference between the groups was not significant.
Both evaluated populations were comparable in their composition. In terms of median age, and in terms of the proportion of patients previously taking analgesics, there were no statistically significant differences between the populations. The characteristics of the population are schematically shown in Table $1 \mathrm{~b}$, which shows the number of patients with/or without analgesic treatment before entering the clinical trial.

Regarding the assessment of mobility by the measurement of the Thomayer distance (Table 2a), both treatments showed improvement in mobility (statistically significant reduction of the distance) during the treatment. This improvement was statistically significant from the baseline in both groups and there were no statistically significant differences between the treatment groups, although slightly better results were achieved in the group with the MD applications. No progress was observed regarding the Lassegue test values (also presented in Table $2 b$ ) during the treatment.

Table 2a. Thomayer distance.

Item

(MD inj./Mesocaine inj.)

MD inj.

Mesocaine inj.

\begin{tabular}{|c|c|c|c|}
\hline Thomayer distance (cm), Visit 1 & & $12.2 \pm 10.0$ & $13.1 \pm 9.16$ \\
\hline Thomayer distance (cm), Visit 3 & & $11.3 \pm 9.64$ & $11.0 \pm 6.86$ \\
\hline Thomayer distance (cm), Visit 6 & & $8.07 \pm 9.50 *$ & $9.42 \pm 8.29 *$ \\
\hline Thomayer distance (cm), Visit 7 & & & $10.0 \pm 7.52 *$ \\
\hline
\end{tabular}

* - statistically significant difference from baseline $\mathrm{p}<0.05$ (MANOVA and post hoc Fisher's LSD test). The difference between the groups was not significant.

Table 2b. Lassegue test.

\begin{tabular}{lcll}
\hline Item & $\begin{array}{c}\text { n } \\
\text { (MD inj./Mesocaine inj.) }\end{array}$ & MD inj. & Mesocaine inj. \\
\hline $\begin{array}{l}\text { Lassegue test } \leq 45^{\circ} \text {, Visit } 1 \\
\text { Lassegue test } \leq 45^{\circ} \text {, Visit } 3\end{array}$ & & Negative 73 times & Positive 1 time \\
Lassegue test $\leq 45^{\circ}$, Visit 6 & $73 / 24$ & Negative 73 times & Negative 23 times \\
Lassegue test $\leq 45^{\circ}$, Visit 7 & & Negative 73 times & Negative 24 times \\
& & Negative 73 times & Negative 24 times
\end{tabular}

The principal endpoints of the study included back pain assessed by patients using a visual analogue scale (VAS) (Tables 3a and 3b). Exertional and rest pain gradually decreased. There was no statistically significant difference between the study groups regarding the pain relief and its outcome. A statistically significant decrease in exercise-induced pain from baseline was achieved in both groups from Visit 3 and statistically significant difference in rest pain was also recorded in the group treated with MD products from Visit 3 and in the group of patients treated with trimecaine from Visit 4 . The difference between the treatment groups could be 
attributed to the rather low number of patients treated in the trimecaine group. Another evaluated parameter was the consumption of the auxiliary analgesic (paracetamol) in the reference period (Table 4). As shown by the results, the consumption of paracetamol was significantly lower in the group treated with MD preparations (approximately half) than in the group treated with Mesocaine. The difference is statistically and clinically significant $(\mathrm{p}=0.0485)$ in favor of MD injections.

Table 3a. Visual analogue scale for exertional pain (VAS), absolute values (cm), assessed by the patient.

\begin{tabular}{|c|c|c|c|c|}
\hline Item & $\frac{n}{(M D \text { inj./Mesocaine inj.) }}$ & MD inj. & Stat. & Mesocaine inj. \\
\hline$V A S$, Exertional pain, Visit 1 & & $67.0 \pm 12.6$ & NS & $70.5 \pm 12.3$ \\
\hline VAS, Exertional pain, Visit 2 & & $63.8 \pm 12.1$ & NS & $68.2 \pm 17.3$ \\
\hline VAS, Exertional pain, Visit 3 & & $58.4 \pm 15.1^{*}$ & NS & $62.0 \pm 20.4^{*}$ \\
\hline VAS, Exertional pain, Visit 4 & $73 / 24$ & $53.7 \pm 18.0 *$ & NS & $55.2 \pm 24.4^{*}$ \\
\hline$V A S$, Exertional pain, Visit 5 & & $43.5 \pm 20.1^{*}$ & NS & $42.9 \pm 26.1^{*}$ \\
\hline$V A S$, Exertional pain, Visit 6 & & $37.9 \pm 22.3^{*}$ & NS & $39.3 \pm 26.1^{*}$ \\
\hline$V A S$, Exertional pain, Visit 7 & & $34.1 \pm 22.1 *$ & NS & $37.3 \pm 29.0^{*}$ \\
\hline
\end{tabular}

* - statistically significant difference from baseline $\mathrm{p}<0.05$ (MANOVA and post hoc Fisher's LSD test). The differences between groups were not statistically significant.

Table 3b. Visual analogue scale for rest pain (VAS), absolute values (cm), assessed by the patient.

\begin{tabular}{|c|c|c|c|c|}
\hline Item & $\begin{array}{c}n \\
\text { (MD inj./Mesocaine inj.) }\end{array}$ & MD inj. & Stat. & Mesocaine inj. \\
\hline VAS, Rest pain, Visit 1 & & $59.0 \pm 14.7$ & NS & $59.2 \pm 15.1$ \\
\hline VAS, Rest pain, Visit 2 & & $59.2 \pm 15.2$ & NS & $62.9 \pm 18.8$ \\
\hline VAS, Rest pain, Visit 3 & & $52.3 \pm 19.0^{*}$ & NS & $51.1 \pm 19.4$ \\
\hline$V A S$, Rest pain, Visit 4 & $73 / 24$ & $45.7 \pm 19.2^{*}$ & NS & $46.2 \pm 22.2 *$ \\
\hline VAS, Rest pain, Visit 5 & & $36.1 \pm 20.2 *$ & $\mathrm{NS}$ & $33.3 \pm 24.1 *$ \\
\hline$V A S$, Rest pain, Visit 6 & & $31.5 \pm 23.1^{*}$ & NS & $31.7 \pm 25.2 *$ \\
\hline$V A S$, Rest pain, Visit 7 & & $27.1 \pm 21.9^{*}$ & NS & $30.8 \pm 30.0^{*}$ \\
\hline
\end{tabular}

* - statistically significant difference from baseline $p<0.05$ (MANOVA and post hoc Fisher's LSD test). The differences between groups were not statistically significant.

Table 4. Paracetamol consumption during Visits 1-6 (number of tablets).

\begin{tabular}{ccc}
$\begin{array}{c}\text { MD inj. } \\
(\mathbf{n}=\mathbf{7 3})\end{array}$ & $\begin{array}{c}\text { Mesocaine inj. } \\
(\mathbf{n}=\mathbf{2 4})\end{array}$ & $\mathbf{p}$ \\
\hline $13.7 \pm 20.7$ & $24.5 \pm 28.9$ & $\begin{array}{c}\text { One-tailed T-test } \\
*(\mathrm{p}=0.0485)\end{array}$ \\
\hline
\end{tabular}

* - statistically significant difference between groups.

Tables $5 \mathrm{a}$ and $5 \mathrm{~b}$ show treatment tolerability assessed by the patients (evaluation during Visits 6 and 7). Assessments of "very good tolerability" and "good tolerability" prevailed in both groups, in both intervals. The patients in both groups rated the tolerability of the treatment similarly at Visit 6; patients treated with MD products assessed the treatment tolerability slightly better at Visit 7. The assessment of tolerability decreased at Visit 7, whereas in the group treated with trimecaine tolerability decreased at Visit 6 .

Table 6 shows the assessment of quality of life using the EuroQol questionnaire (the patient's previous health condition versus "today's" health condition at the reference control examination). The condition 
Table 5a. Treatment tolerability (assessed by the patient) during Visit 6.

\begin{tabular}{lcc}
\hline Category & MD inj. & Mesocaine inj. \\
\hline Very good & $50(68.5 \%)$ & $17(70.8 \%)$ \\
Good & $15(20.5 \%)$ & $4(16.7 \%)$ \\
Medium & $8(11.0 \%)$ & $3(12.5 \%)$ \\
Poor & 0 & 0 \\
\hline
\end{tabular}

improvement from baseline is evident in both groups, with no statistically significant differences between the groups found.

Table 7 shows the selected results of the OSWESTRY questionnaire assessment, which expresses the degree of disability. The table shows improvement of
Table 5b. Treatment tolerability (assessed by patients) during Visit 7.

\begin{tabular}{|c|c|c|}
\hline Item & MD inj. & Mesocaine inj. \\
\hline Very good & $51(69.9 \%)$ & $14(58.3 \%)$ \\
\hline Good & $17(23.3 \%)$ & $8(33.3 \%)$ \\
\hline Medium & $4(5.48 \%)$ & $2(8.33 \%)$ \\
\hline Poor & $1(1.37 \%)$ & 0 \\
\hline
\end{tabular}

the patients' condition in both groups during the treatment with a slight tendency toward better results in the group with MD injections (mostly without a statistically significant difference between treatment groups).

Table 6. Quality of life questionnaire (EuroQol).

\begin{tabular}{|c|c|c|c|c|c|c|c|c|c|}
\hline $\begin{array}{l}\text { In } \\
\text { comparison } \\
\text { with the } \\
\text { health }\end{array}$ & & Treatment & $\begin{array}{c}n \\
\text { (MD inj./ } \\
\text { Mesocaine } \\
\text { inj.) }\end{array}$ & $\begin{array}{c}2^{\text {nd }} \text { Visit } \\
\text { (Frequency, } \\
\% \text { ) }\end{array}$ & $\mathbf{p}$ & $\begin{array}{c}6^{\text {th }} \text { Visit } \\
\text { (Frequency, } \\
\% \text { ) }\end{array}$ & $\mathbf{p}$ & $\begin{array}{c}7^{\text {th }} \text { Visit } \\
\text { (Frequency, } \\
\% \text { ) }\end{array}$ & $\mathbf{p}$ \\
\hline in the last & \multirow{2}{*}{ Better } & MD inj. & \multirow{6}{*}{$73 / 24$} & $3(4.11 \%)$ & \multirow{6}{*}{ n. s. } & $43(58.9 \%)$ & & $44(60.3 \%)$ & \multirow{6}{*}{ n. s. } \\
\hline 12 months, & & Mesocaine inj. & & $2(8.33 \%)$ & & $13(54.2 \%)$ & & $15(62.5 \%)$ & \\
\hline my health & Nearly & MD inj. & & $39(53.4 \%)$ & & $23(31.5 \%)$ & & $23(31.5 \%)$ & \\
\hline condition & the same & Mesocaine inj. & & $9(37.5 \%)$ & & $9(37.5 \%)$ & n. s. & $6(25.0 \%)$ & \\
\hline today is: & \multirow{2}{*}{ Worse } & MD inj. & & $31(42.5 \%)$ & & $7(9.59 \%)$ & & $6(8.22 \%)$ & \\
\hline & & Mesocaine inj. & & $13(54.2 \%)$ & & $2(8.33 \%)$ & & $3(12.5 \%)$ & \\
\hline
\end{tabular}

Table 7. OSWESTRY questionnaire (Oswestry Disability Index).

\begin{tabular}{lllllllll}
\hline Item & Treatment & $\begin{array}{c}\mathbf{n} \\
\text { (MD inj./ } \\
\text { Mesocaine inj.) }\end{array}$ & $\mathbf{2}^{\text {nd }}$ Visit & $\mathbf{p}$ & $\mathbf{6}^{\text {th }}$ Visit & p & $7^{\text {th }}$ Visit & p \\
\hline $\begin{array}{l}\text { Disability } \\
(\%)\end{array}$ & MD inj. & $73 / 24$ & $27.5 \pm 13.5$ & 0.217 & $19.0 \pm 13.9$ & $0.214^{*}$ & $17.6 \pm 13.5$ & $0.310^{*}$ \\
\hline
\end{tabular}

Statistical significance of the difference, parametric and non-parametric evaluations. Parametric evaluation - the statistical significance of the difference between both drugs in a given interval for each item (MANOVA and post hoc Fisher's LSD test, MF). Non-parametric evaluation - in the type of disability according to the percent, the difference between the drugs is evaluated by chi-square test $(*-$ statistical significance, $p<0.05)$.

\section{Discussion}

In our study, we evaluated the efficacy and safety of combined products containing collagen which were subcutaneously administered (MD-Lumbar, MD-Neural, MD-Muscle, Guna, Milano, Italy) for the treatment of patients with acute non-specific back pain. These MD-products represent a new concept in the 
treatment of pain, based on strengthening the collagen matrix underlying the musculoskeletal system structures (the so called bioscaffold) and on the analgesic and antioxidative effects of these products. We compared the effects of the MD products with standard medicine, used for this indication, trimecaine (product Mesocaine $1 \%$ ) also in subcutaneous application. When the MD product is applied, a temporary porous collagen matrix forms in the tissue and the active substances are gradually released into the target area at a defined speed, thus ensuring their prolonged activity.

Collagen is the primary active ingredient of the MD products. The effect of locally applied collagen administration is structural and functional. The collagen is delivered directly to the areas where it is lacking and by creating so called adhesive barrier, it strengthens, gives structure and protects muscles, tendons and ligaments. It improves the profile of the collagen fibres and consequently, all collagen-containing anatomical structures. In addition, locally administered collagen helps to release painful muscle tension in the region and to restore its physiological function. Although the prompt analgesic activity of the MD products was not primarily expected, their administration removed the cause of pain. This may explain why their efficacy is comparable to other analgesic medications, although their analgesic effect is achieved by non-pharmacological means.

This study also has its limitations. The most important limitation is the absence of a placebo group in the study. This may be particularly important in diseases such as acute LBP, which usually have a limited duration (self-limited). However, the administration of placebo in this case also presents ethical problems.

There is lack of published data on injectable collagen in the treatment of back pain. Nevertheless, there is one randomized clinical study (Nitecka-Buchta et al. 2018) demonstrating the effectiveness of intramuscularly administered collagen in patients suffering from myofascial pain. The use of collagen was even superior to intramuscular administration of lidocaine. Additionally, the intraarticular injection of MD-preparations containing collagen exerted similar clinical effects as sodium hyaluronate in patients with knee osteoarthritis (Martin Martin et al. 2016).

Importantly, even the effectiveness of common clinically used injections with local anesthetics, either alone or in combination with corticosteroids remains disputable (Manchikanti et al. 2016) and is mostly based on empirical data. Some authors also mention the so-called "needle effect", i.e. an observable beneficial effect caused by puncture with a needle only. Hence, the recorded effect in our study might be at least partially attributed to needle stimulation of some dermal/subdermal structures as previously reported in a meta-analysis by Manheimer et al. (2007). They concluded that acupuncture seems to have a genuine biological effect, as suggested by the small short-term improvements in pain and function compared to placebo in the treatment of osteoarthritis of the knee. On the contrary, the effectiveness of acupuncture alone in the treatment of LBP has recently been the subject of re-evaluation by NICE (2016) who have reported that acupuncture is no longer recommended for the management of LBP with or without sciatica. Therefore, the beneficial effects of the active pharmaceutical ingredients in the MD injections have been suggested as the principal explanation of the recorded effects.

Importantly, the good safety profile of the MD products are very valuable, especially in comparison to the commonly used NSAIDs, which possess a huge risk of gastrotoxicity, hepatotoxicity, cardiotoxicity and nephrotoxicity, namely after long-term use and/or the use of high daily doses. Therefore, locally applied treatment with collagen products represents a safer treatment option.

\section{Conclusions}

The study results showed that in most evaluated efficacy parameters (e.g. pain assessed by visual analogue scale, functional parameters, questionnaires focusing on disability and quality of life):

- the efficacy of both treatments is comparable both in terms of quantification of the effect and its time course;

- the MD products are relatively without any risk of adverse effects;

- the MD product analgesic effect persisted even through follow-up examinations;

- a statistically and clinically significant difference in the consumption of paracetamol (as a system analgesic) was observed - approximately twice in treatment group B (trimecaine) compared to treatment group A (MD products), i.e. to achieve the same effect, significantly more rescue medications were consumed;

- the tolerability was very good or good in both groups, but was statistically better in the MD products group. 
Finally, the study results can be assessed by noting that in patients with acute nonspecific back pain, collagen-containing combination products (i.e. MD-Lumbar, MD-Neural, MD-Muscle) have a comparable efficacy with the standardly used trimecaine; the advantages of these products include lower consumption of supplemental analgesics and better tolerability. According to the results of this study, these products could properly extend the current relatively limited range of treatment methods available for acute non-specific back pain.

\section{Conflict of Interest}

There is no conflict of interest.

\section{Acknowledgements}

This work was supported by PROGRESS Q 35.

\section{References}

BHANGARE KP, KAYE AD, KNEZEVIC NN, CANDIDO KD, URMAN RD: An analysis of new approaches and drug formulations for treatment of chronic low back pain. Anesthesiol Clin 35: 341-350, 2017.

CHARNEUX L, DEMOULIN C, VANDERTHOMMENT M, TOMASELLA M, FERRARA MA, GROSDENT S, BETHLEN S, FONTAINE R, GILLET P, RACARU T, KAUX JF: Platelet-rich plasma (PRP) and disc lesions: a review of the literature (in French). Neurochirurgie 63: 473-477, 2017.

FURLAN AD, VAN TULDER M, CHERKIN D, TSUKAYAMA H, LAO L, KOES B, BERMAN B: Acupuncture and dry-needling for low back pain: an updated systematic review within the framework of the Cochrane collaboration. Spine 30: 944-963, 2005.

ITOH K, KATSUMI Y, KITAKOJI H: Trigger point acupuncture treatment of chronic low back pain in elderly patients - a blinded RCT. Acupunct Med 22: 170-177, 2004.

KNEZEVIC NN, MANDALIA S, RAASCH J, KNEZEVIC I, CANDIDO KD: Treatment of chronic low back pain new approaches on the horizon. J Pain Res 10: 1111-1123, 2017.

LIU D, SUN M, XU D, MA X, GAO D, YU H: Inhibition of TRPA1 and IL-6 signal alleviates neuropathic pain following chemotherapeutic bortezomib. Physiol Res 68: 845-855, 2019.

MANCHIKANTI L, HIRSCH JA, FALCO FJ, BOSWELL MV: Management of lumbar zygapophysial (facet) joint pain. World J Orthop 7: 315-337, 2016.

MANHEIMER E, LINDE K, LAO L, BOUTER LM, BERMAN BM: Meta-analysis: acupuncture for osteoarthritis of the knee. Ann Intern Med 146: 868-877, 2007.

MARTIN MARTIN LS, MASSAFRA U, BIZZI E, MIGLIORE A: A double blind randomized active-controlled clinical trial on the intra-articular use of MD-Knee versus sodium hyaluronate in patients with knee osteoarthritis ("Joint"). BMC Musculoskelet Disord 17: 94, 2016.

MILANI L: A new and refined injectable treatment for musculoskeletal disorders. Bioscaffold properties of collagen and its clinical use. Physiol Reg Med 5: 3-15, 2010.

NICE: Low back pain and sciatica in over 16s: assessment and management. NICE guideline NG 59, 2016. https://www.nice.org.uk/guidance/NG59 (accessed 11 June 2019).

NITECKA-BUCHTA A, WALCZYNSKA-DRAGON K, BATKO-KAPUSTECKA J, WIECKIEWICZ M: Comparison between collagen and lidocaine intramuscular injections in terms of their efficiency in decreasing myofascial pain within masseter muscles: a randomized, single-blind controlled trial. Pain Res Manag 2018: $8261090,2018$.

PAVELKA K, JAROSOVA H, SLEGLOVA O, SVOBODOVA R, VOTAVOVA M, MILANI L, PROCHAZKA Z, KOTLAROVA L, KOSTIUK P, SLIVA J, MERONI AM: Chronic low back pain: current pharmacotherapeutic therapies and a new biological approach. Curr Med Chem 26: 1019-1026, 2019.

RANDELLI F, MENON A, GIAI VIA A, MAZZOLENI MG, SCIANCALEPORE F, BRIOSCHI M, GAGLIANO N: Effect of a collagen-based compound on morpho-functional properties of cultured human tenocytes. Cells 7: E246, 2018. 
VAN TULDER M, BECKER A, BEKKERING T, BREEN A, DEL REAL BT, HUTCHINSON A, KOES B, LAERUM E, MALMIVAARA A: Chapter 3. European guidelines for the management of acute nonspecific low back pain in primary care. Eur Spine J15 (Suppl 2): S169-S191, 2006.

WEBB MP, HELANDER EM, MENARD BL, URMAN RD, KAYE AD: Tanezumab: a selective humanized mAb for chronic lower back pain. Ther Clin Risk Manag 14: 361-367, 2018.

YAMAMOTOVA A, HRABAK P, HRIBEK P, ROKYTA R: Do multiple body modifications alter pain threshold? Physiol Res 66 (Suppl 4): S493-S500, 2017.

ZHANG Y, GAO Y, LI CY, DONG W, DONG Y, LI MN, LIU YN, XU SL: Galanin receptor 1 plays an antinociceptive effect via inhibiting PKA activation in the nucleus accumbens of rats with neuropathic pain. Physiol Res 68: 511-518, 2019. 